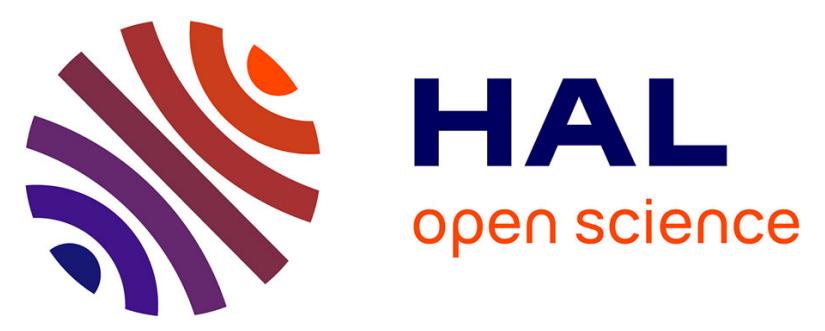

\title{
Enseignement et apprentissage des mathématiques à l'école primaire dans un contexte bilingue breton-francais
}

Caroline Poisard, Martine Kervran, Erwan Le Pipec, Stéphane Alliot, Ghislaine Gueudet, Hélène Hili, Nathalie Jeudy-Karakoç, Gwenole Larvol

\section{To cite this version:}

Caroline Poisard, Martine Kervran, Erwan Le Pipec, Stéphane Alliot, Ghislaine Gueudet, et al.. Enseignement et apprentissage des mathématiques à l'école primaire dans un contexte bilingue bretonfrancais. Spirale - Revue de Recherches en Éducation, 2014, 54, pp.129-150. hal-01067694

\section{HAL Id: hal-01067694 https://hal.science/hal-01067694}

Submitted on 26 Sep 2014

HAL is a multi-disciplinary open access archive for the deposit and dissemination of scientific research documents, whether they are published or not. The documents may come from teaching and research institutions in France or abroad, or from public or private research centers.
L'archive ouverte pluridisciplinaire HAL, est destinée au dépôt et à la diffusion de documents scientifiques de niveau recherche, publiés ou non, émanant des établissements d'enseignement et de recherche français ou étrangers, des laboratoires publics ou privés. 


\title{
Enseignement et apprentissage des mathématiques à l'école primaire dans un contexte bilingue breton-français
}

Revue Spirale 54, 2014

\author{
Auteurs \\ Caroline Poisard, ESPE de Bretagne/UBO, CREAD, caroline.poisard@espe-bretagne.fr \\ Martine Kervran, ESPE de Bretagne/UBO, CREAD \\ Erwan Le Pipec, ESPE de Bretagne/UBO, CRBC \\ Stéphane Alliot, DSDEN des Côtes-d'Armor \\ Ghislaine Gueudet, ESPE de Bretagne/UBO, CREAD, ghislaine.gueudet@espe-bretagne.fr \\ Hélène Hili, ESPE de Bretagne/UBO \\ Nathalie Jeudy-Karakoç, Circonscription Quimper Sud, Université du Maine \\ Gwenole Larvol, ESPE de Bretagne/UBO, CREAD
}

\section{Résumé :}

Cet article concerne l'enseignement des mathématiques en breton à l'école primaire. Nous nous référons en particulier aux travaux de Adler $(2000,2001)$, qui invitent à considérer la pluralité de langues non seulement comme une difficulté, mais aussi comme une ressource possible pour l'enseignement des mathématiques. Ils invitent également à porter attention aux ressources matérielles disponibles, et à leur usage par les professeurs. Nous étudions les caractéristiques de la langue bretonne susceptibles d'amener celle-ci à constituer, ou non, une ressource pour l'enseignement des mathématiques. A partir des réponses de professeurs à un questionnaire, nous étudions les ressources matérielles qu'ils utilisent, et celles qui leur manquent. Enfin, nous discutons nos premiers résultats et essayons de proposer quelques pistes pour une prise en compte de la spécificité de la langue et de la culture bretonne dans l'enseignement des mathématiques en breton.

\section{Abstract :}

This article deals with teaching mathematics in Breton at primary school. We mainly refer to Adler's research $(2000,2001)$ inviting to consider linguistic plurality not only as a difficulty, but also as a possible resource for mathematics teaching. Her work invites to focus on available material resources and on the way teachers use them. We study the characteristic features of Breton liable to constitute - or not - the language as a resource for mathematics teaching. Relying on teachers' answers to a questionnaire, we also study the material resources they use and what resources are lacking. We finally discuss our first results and try to suggest a few trails towards taking the linguistic and cultural specificities of the language into account in mathematics teaching.

Mots-clefs : didactique des mathématiques, bilinguisme, école primaire, formation des professeurs, langue bretonne, manuel scolaire, ressources, terminologie mathématique en breton, numération orale

Key words : Breton language, Breton terminology for mathematics, didactics, mathematics education, multilingualism, primary school, resources, teacher education, textbooks, oral numeration 
Le travail présenté dans cet article concerne l'enseignement des mathématiques dans un contexte d'enseignement bilingue breton-français, plus précisément au niveau de l'école primaire. Il a été effectué dans le cadre d'un groupe de recherche de l'ESPE de Bretagne, intitulé "Mathématiques en Langue Bretonne à L'Ecole Aujourd'hui ${ }^{1}$ » (MELBA), et associant des chercheurs en didactique des mathématiques, en didactique des langues, en linguistique, et des enseignants d'école primaire. La première partie de cet article situe brièvement le contexte de l'enseignement bilingue breton-français, plus particulièrement en ce qui concerne les mathématiques. Dans la deuxième partie, nous présentons des travaux en lien avec notre thème d'étude, le cadre théorique que nous retenons, nos questions de recherche et la méthodologie mise en œuvre. La troisième partie est consacrée à l'analyse de certaines spécificités de la langue bretonne et de leurs conséquences potentielles, pour l'apprentissage des mathématiques. La quatrième partie concerne le point de vue des professeurs, tel qu'il ressort de l'analyse de réponses à un questionnaire élaboré et diffusé par le groupe.

\section{L'enseignement des mathématiques en langue bretonne : éléments de contexte}

La société civile bretonne est clairement aujourd'hui une société très majoritairement francophone. Selon les données numériques de 2010, parmi les 4500000 habitants de la région Bretagne, seuls 205000 sont brittophones (4,5\%) (Broudic 2009). De plus, la plupart de ces locuteurs sont âgés ; certaines projections indiquent qu'à l'horizon 2040, le nombre de brittophones pourrait être réduit à 60000 (1,3\%) (Le Pipec 2014). Pourtant la société bretonne n'a pas renoncé à son bilinguisme passé, qu'il n'est donc pas tant question de maintenir, que de restaurer (Le Pipec 2014). C'est dans cette perspective que s'inscrivent les choix politiques régionaux de soutien à la langue bretonne (figure 1), en particulier le développement de l'enseignement bilingue qui est au cœur de notre étude.

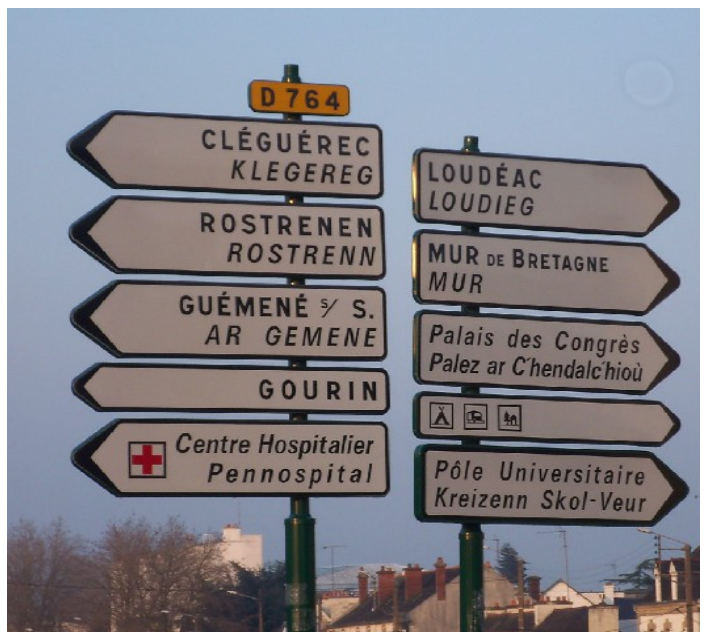

Figure 1. Le bilinguisme, un enjeu sociétal en Bretagne.

L'enseignement bilingue breton-français existe sous la forme de trois filières : les écoles privées associatives Diwan (le germe) depuis 1977 ; les classes bilingues, au sein des écoles publiques depuis 1983 et des écoles privées catholiques depuis 1990. A la rentrée 2012, sur les cinq départements de la Bretagne historique ${ }^{2}$, ces différentes filières scolarisaient un total de 14706 élèves de la maternelle au lycée (Rapport 2013 de l'Office Public de la Langue Bretonne OPLB). Pour nos travaux, nous considérons seulement le cas du premier degré, qui rassemble le plus grand nombre d'élèves (6 257 en élémentaire et 6003 en pré-élementaire à la rentrée 2012, soit 12 260).

1 Projet soutenu par la maison des sciences de l'homme de Bretagne et la Région Bretagne.

2 Ainsi qu'à Paris, où une école Diwan scolarise 53 élèves. 
Deux grands modèles pédagogiques prévalent, l'un dit immersif où l'enseignement est intégralement ou majoritairement en breton (Diwan, privé associatif), l'autre dit paritaire où la parité horaire de l'utilisation des langues est prévue (public et privé catholique). Dans les écoles Diwan, en cycle 1, c'est uniquement en breton que se déroulent les activités de classe, le français est introduit progressivement à partir du cycle 2 mais les enseignements se déroulent majoritairement en breton. D'autre part, dans les écoles paritaires, les textes officiels précisent que le breton ne peut dépasser la moitié du temps scolaire. Nous parlerons cependant dans tous les cas d'enseignement bilingue, car les deux langues sont nécessairement présentes dans l'environnement scolaire.

Comme le montrent les chiffres que nous avons évoqués ci-dessus, l'enseignement bilingue breton-français revêt une allure de reconquête et de défi collectif. Alors que des parents de plus en plus nombreux choisissent d'inscrire leurs enfants dans des écoles bilingues, l'une des difficultés qui se présentent est le recrutement d'un nombre suffisant d'enseignants pratiquant la langue bretonne. Des centres d'enseignement de la langue pour un public adulte se sont développés; on se représente bien, cependant, le défi que constitue un enseignement en breton pour un professeur ayant lui-même appris la langue de fraîche date. Notons que, de plus, dans cet enseignement bilingue, la plupart des classes sont multi-niveaux (les classes comportant au moins trois niveaux sont même fréquentes), ce qui complexifie le travail de l'enseignant.

La langue bretonne a été modernisée, en vue de son enseignement (Kergoat 2012). Ces travaux de modernisation ont débuté à la charnière entre $\mathrm{XIX}^{\mathrm{e}}$ et $\mathrm{XX}^{\mathrm{e}}$ siècle. Cependant, ce n'est que vers 1980 qu'une véritable demande va exister et aboutir à l'entrée à l'école primaire des premiers élèves de Diwan (en 1977). Aujourd'hui l'enseignement bilingue est dispensé de la maternelle jusqu'au baccalauréat. Dans un premier temps, les terminologies en usage dans l'enseignement vont donc refléter des choix anciens, où dominait une stratégie de distanciation avec le français. D'où la recherche de "pureté lexicale " et de "celticité » maximale. Mais les années 1980 à 1990 voient également l'émergence de nouveaux choix terminologiques, favorisant la prise en compte de racines internationales (c'est-à-dire grecques et latines). Au-delà des choix officiels, une multiplicité de profils linguistiques existe chez les professeurs, qui peuvent privilégier différentes variétés de breton, plutôt dialectal ou plutôt littéraire selon leur propre pratique de la langue, pratique dans laquelle le français peut avoir une influence plus ou moins importante. Nous reviendrons sur ces aspects et sur leurs conséquences en mathématiques dans la partie 3 de cet article.

Du point de vue des supports disponibles pour l'enseignement des mathématiques, ceux-ci sont publiés par une maison d'édition spécialisée, Ti-embann ar skolioù (La maison d'édition des écoles, notée TES $^{3}$ par la suite). Il existe un glossaire couvrant le programme de l'école primaire, et celui des classes de sixième et cinquième (Geriaoueg matematik); nous nous référerons ici à deux éditions successives de ce glossaire, datant respectivement de 1998 et de 2005. Il existe par ailleurs des manuels traduits du français : les traductions de Objectif Calcul (Hatier) du CP au CM2 ont été éditées entre 1994 et 1998 ; celles de J'apprends les maths (Retz) du CP au CM2 entre 2001 et 2005 ; et enfin Cap Maths (Hatier) du CP au CM2 depuis 2010, les dernières traductions étant prévues pour 2014. Seuls les manuels élèves ont été traduits, les professeurs utilisent le livre du maître associé en français. Même si dans les derniers manuels traduits, on peut trouver quelques références à la culture bretonne, les traductions sont en grande majorité des traductions littérales du français en breton sans réelle prise en compte de la culture bretonne.

Nous allons maintenant nous pencher sur des travaux en lien avec le thème de nos recherches.

3 TES est une antenne éditoriale dédiée à la langue bretonne, rattachée au CRDP (Centre régional de documentation pédagogiques) de Rennes, depuis 1993. Site Internet http://www.cndp.fr/crdprennes/crdp/services/tes/index.php/ 


\section{Bilinguisme, enseignement des mathématiques et ressources}

\subsection{Enseignement bilingue et recherche en didactique des mathématiques}

Notre étude est la première à considérer la question de l'enseignement des mathématiques en breton avec un point de vue didactique. Cependant de nombreux travaux existent à propos de l'enseignement bilingue, notamment en mathématiques, et des questions soulevées par cet enseignement: en effet le monolinguisme qui semble naturel en France est loin d'être majoritaire au niveau international, notamment parce que de nombreux pays possèdent plusieurs langues officielles.

Les travaux menés en didactique des langues sur les effets de l'enseignement bi-plurilingue soulignent que celui-ci est susceptible de favoriser la conceptualisation. Les recherches anglosaxonnes sur l'intégration des langues dans les autres champs disciplinaires (Cummins 1984, 2001 ; Swain 1996) comme les travaux francophones (notamment Gajo 2001) concluent à l'existence d'un atout bilingue pour la conceptualisation dans les disciplines dites non linguistiques (Gajo 2007). L'approche des concepts selon une pluralité de points de vue linguistiques et culturels favorise leur compréhension. Les enjeux langagiers vont de pair avec les enjeux disciplinaires, qu'il s'agisse de la dimension lexicale (variété des traductions des termes savants; pluralité des (re)formulations), des aspects grammaticaux (fonction des connecteurs par exemple) ou des particularités textuelles (par exemple: hypothèses, proposition, argumentation, démonstration, etc.). La pluralité langagière constitue « un outil naturel d'élaboration et d'accès au savoir » (Gajo 2007, 37) et permet de clarifier simultanément la terminologie et l'objet de savoir disciplinaire qu'elle recouvre.

En ce qui concerne plus particulièrement les mathématiques, plusieurs études sur l'enseignement et l'apprentissage des mathématiques en contexte bilingue ont analysé plus précisément les raisons pour lesquelles l'utilisation de deux langues pouvait favoriser les apprentissages. Les travaux menés en Irlande par Ní Ríordáin (Ní Ríordáin 2013, Ní Ríordáin, \& McCluskey 2012) dans un contexte proche de celui de notre étude partent du constat de meilleures performances en mathématiques des élèves bilingues. Retenant une perspective de théorie de l'activité (Vygotsky 1962), Ní Ríordáin considère la langue comme un artefact culturel, jouant un rôle essentiel de médiation dans l'apprentissage des mathématiques. Elle montre que le gaëlique est porteur de spécificités susceptibles de rendre cette médiation facilitatrice dans l'apprentissage des mathématiques. Ainsi la structure des phrases en gaëlique met en évidence les informations essentielles, ce qui peut favoriser l'interprétation d'un énoncé de problème; par ailleurs, certains mots peuvent avoir plusieurs sens, selon le contexte, ce qui développe l'attention des élèves au contexte, y compris dans le cas de problèmes posés en anglais. Elle souligne toutefois que les différences de performances peuvent aussi provenir de facteurs sociaux (Setati \& Planas 2012), les parents des élèves de l'enseignement bilingue appartenant à des catégories socioprofessionnelles plus favorisées.

Moschkovich (2002), qui étudie l'apprentissage des mathématiques aux Etats-Unis par des élèves dont la langue maternelle n'est pas l'anglais (élèves hispano-américains notamment), montre elle aussi que le bilinguisme peut être une ressource si les conditions d'enseignement permettent aux élèves une véritable participation dans les pratiques discursives mathématiques de la classe. Il ne s'agit pas pour ces élèves d'apprendre seulement du vocabulaire mathématique, mais de participer à un discours qui dépasse le langage, et intègre des gestes, des pratiques partagées. Ceci est possible lorsque les enseignants sont suffisamment avertis des spécificités linguistiques et culturelles de leurs élèves, et tiennent compte de celles-ci pour aménager leur enseignement (Moschkovich \& Nelson-Barber 2009). En ce qui concerne les pays où l'enseignement se fait en français, Mohamed-Sagayar (2007) montre par exemple qu'au Niger, où coexistent cinq langues principales, les professeurs ont recours aux langues familiales des élèves pour l'enseignement de la numération. Par exemple, 
en langue hausa 15 se dit goma sha biyar, soit littéralement « 10 et 5 », ce qui permet un accès beaucoup plus direct au principe sous-jacent de numération entière que le terme français quinze. Ce point, concernant les irrégularités à l'oral de la numération entière en français, est une difficulté connue (Brousseau 2012); cet aspect de numération entière joue un rôle essentiel dans notre travail (partie 3). Au-delà des nombres entiers, des questions semblables se posent pour les fractions, qui retiennent aussi notre attention : ainsi Poisard et Barton (2007), comparant l'anglais et le français, identifient des différences (avec en particulier en anglais l'usage de nombres mixtes, comme $5 \frac{1}{2}$ ) pouvant avoir des conséquences sur les apprentissages, notamment en ce qui concerne les opérations.

De nombreux travaux ont été également menés en Nouvelle-Zélande (Barton et alii 1998, Barton 2008, Meaney et alii 2007) à propos de l'enseignement des mathématiques en maori. Nous retenons en particulier les réflexions concernant les choix de terminologie mathématique. Selon quels critères ces choix doivent-ils être faits, lorsque l'on ne dispose d'aucune traduction historique, pour un terme spécialisé ? Barton et alii (1998) analysent très finement différents les choix qui ont pu être faits en maori, allant de la translittération (correspondance lettre à lettre) à la recherche de métaphores (rere, indiquant un cours d'eau, étant ainsi retenu pour désigner la continuité au sens mathématique). Nous nous pencherons de même sur certains choix de terminologie bretonne qui aujourd'hui encore font débat.

Cependant, dans notre travail la référence essentielle, qui constitue également le cadre théorique que nous mobilisons, provient de travaux de Adler $(2000,2001)$.

\subsection{Conceptualisation des ressources et questions de recherche}

Les travaux de Adler se placent dans le contexte de l'Afrique du Sud post-apartheid, où le développement de l'éducation, dans certaines régions particulièrement défavorisées, est un défi sociétal important. Alors que les professeurs expriment un manque de ressources matérielles, Adler propose une nouvelle conceptualisation de la notion de ressource. Est ainsi vu comme ressource tout ce qui peut ressourcer l'activité du professeur de mathématiques on est proche, là encore, d'une perspective de théorie de l'activité, mettant l'accent sur la médiation réalisée par les ressources. Adler distingue des ressources matérielles (tableau noir, manuel scolaire); des ressources culturelles et des ressources humaines. Parmi les ressources culturelles, elle montre que les langues peuvent constituer des ressources essentielles, dans des classes où sont parfois présentes dix-huit langues différentes. En appui sur un projet de formation continue, organisé sur plusieurs années, Adler montre comment la diversité linguistique peut progressivement être constituée en ressource par le professeur de mathématiques.

Cette perspective est proche de celle de la théorie des situations (Brousseau 1998) : on peut considérer l'ensemble des ressources d'un professeur comme le milieu avec lequel ce professeur interagit (Margolinas 2002). Toutefois nous avons préféré retenir la conceptualisation proposée par Adler, car celle-ci amène à porter une attention particulière à deux dimensions, essentielles dans notre étude. D'une part les potentialités de l'emploi de langues différentes, se constituant en ressources pour l'enseignement des mathématiques. Adler se centre essentiellement sur l'apport d'une réflexion sur les termes, pour la conceptualisation; les travaux de Barton (2008) invitent de plus à considérer les aspects culturels portés par la langue, qui peuvent notamment conférer du sens aux problèmes de mathématiques. D'autre part les ressources susceptibles d'aider le professeur pour cet enseignement. Nous avons souligné que, dans le contexte breton, la maîtrise de la langue pouvait être une difficulté pour les élèves (la proportion d'enfants parlant le breton avant leur entrée à l'école est extrêmement réduite) mais aussi pour certains enseignants. Il est donc essentiel que ces enseignants puissent disposer de ressources adaptées : livres, actions de formation etc. Notre groupe de recherche vise à la fois à analyser la situation actuelle de l'enseignement des mathématiques en langue bretonne, et à contribuer à la production de ressources pour les professeurs (Gueudet \& Trouche 2010), en fonction des ressources 
manquantes éventuellement identifiées (Chevallard \& Cirade 2010).

Ainsi les questions de recherche que nous étudions sont les suivantes :

「Quels sont les atouts et obstacles potentiels de la langue bretonne comme ressource pour l'enseignement et l'apprentissage des mathématiques? Il s'agit d'identifier les spécificités de la langue bretonne qui sont susceptibles d'influencer l'apprentissage des mathématiques dans cette langue, ou dans un contexte bilingue breton-français.

「Est-ce que les ressources matérielles disponibles pour l'enseignement des mathématiques sont susceptibles d'aider les professeurs à exploiter la langue, et la dimension culturelle, pour leur enseignement des mathématiques ?

$\lceil$ Quelles sont les ressources matérielles effectivement utilisées par les professeurs du premier degré pour leur enseignement de mathématiques en langue bretonne ? Est-ce que certaines ressources leur manquent ? Est-ce que les professeurs mobilisent effectivement, dans leurs pratiques, la langue bretonne et les dimensions culturelles comme ressources en mathématiques?

\section{3}

\section{Méthodologie}

Chacune des directions de questionnement ci-dessus a demandé la mise en œuvre d'une méthodologie spécifique.

Pour la question portant sur les potentialités de la langue bretonne, nous avons effectué une analyse du matériau linguistique, dans son rapport et son adéquation aux mathématiques. Ceci nécessite de prendre en compte d'une part la composante endogène de la langue, qui commande par exemple l'expression de la numération orale (noms des nombres) ; mais également le travail sur la langue elle-même, opéré par les linguistes pour la rendre opérationnelle dans l'enseignement : une langue qui a été maintenue longuement en dehors de l'école nécessite en effet d'être dotée d'outils, d'un lexique, d'une phraséologie spécifiques pour servir une telle fin. Et c'est bien ce que montre l'évolution du breton, avec des choix qu'il importe de discuter et d'évaluer.

En ce qui concerne les ressources des professeurs, nous avons tout d'abord recensé et examiné les ressources matérielles existantes. Nous avons ensuite élaboré un questionnaire à destination des enseignants (annexe 1). L'objectif général de ce questionnaire était de faire un état des lieux de la situation actuelle, concernant cinq grandes dimensions de l'enseignement des mathématiques en langue bretonne à l'école :

1. La description des conditions d'enseignement (horaires, effectifs, mais aussi formation reçue pour la langue bretonne, etc.)

2. Le contenu de l'enseignement en breton et ses difficultés (contenus spécialement délicats, causes identifiées, etc.)

3. Les ressources matérielles utilisées

4. Le lien avec l'enseignement des mathématiques en français

5. Les besoins identifiés (en ressources matérielles et en formation)

Ce questionnaire (annexe 1) a été proposé en ligne, avec le soutien d'inspecteurs et de conseillers pédagogiques pour sa diffusion. Nous avons recueilli 80 réponses complètes (pour un effectif d'environ 700 enseignants potentiellement concernés, soit un taux de réponse d'environ $11 \%$ ). Pour analyser les réponses des professeurs, nous nous centrons ici sur les questions portant sur : les spécificités de l'enseignement en breton (question 2), les ressources utilisées par les professeurs (question 3), et les besoins que ceux-ci expriment (question 5). Nous avons croisé les réponses à ces questions avec celles concernant le cycle d'enseignement, l'ancienneté, l'équilibre horaire des deux langues, et le type de formation à la langue bretonne reçu par les professeurs, et effectué des analyses quantitatives simples, calculs de pourcentages, moyennes et écarts-types. 
A la suite de ce questionnaire, nous avons effectué des entretiens et des observations de classe. Nous ne ferons pas référence ici à des analyses systématiques de ces données, qui concernent une phase ultérieure de notre travail (à propos des pratiques effectives), mais nous mentionnerons certaines observations à l'appui de notre étude linguistique.

\section{La langue bretonne, une ressource pour l'apprentissage des mathématiques}

Notre travail concernant un contexte bilingue, il s'agit pour nous avant tout de nous intéresser aux spécificités de la langue bretonne par rapport au français. Ici nous ne pouvons faire un inventaire exhaustif des similitudes et des différences. Nous allons tout d'abord considérer la question essentielle du nom des nombres (numération orale bretonne); puis nous nous pencherons sur certains choix terminologiques qui ont fait, ou font débat, en prenant des exemples en géométrie.

\subsection{Le nom des nombres : numération entière et fractions}

En Nouvelle-Zélande, l'étude de la numération orale maorie ou bien, au Niger, celle de la langue hausa permet une comparaison de l'anglais ou du français vers une langue dont la comptine numérique est régulière, c'est-à-dire dont la modélisation mathématique associée est celle du développement polynomial des nombres en numération de position en base 10. La plupart des langues de l'Asie et du Pacifique possèdent aussi des comptines régulières. Dans l'exemple breton-français, comme nous allons le montrer, c'est l'analyse des deux numérations orales qui nous semble riche pour un enseignement des mathématiques, pour comprendre la numération orale bretonne et la numération orale française ${ }^{4}$. Comme le précise Gajo $(2007,3)$ : « La science s'est développée dans différentes cultures et différentes langues, les savoirs ayant en outre subi diverses migrations. L'école monolingue fait écran à cette réalité, en présentant de manière relativement lisse des savoirs structurés souvent dans un grand nombre de ruptures, y compris linguistiques ». Il poursuit aussi : «L'attention prêtée à l'authentification de la langue, à la densité des savoirs disciplinaires sous-tend le processus de conceptualisation [...] [et] plus généralement, [celui] de clarification » (Ibid, 4).

En base 10, pour exprimer oralement les nombres usuels, il est nécessaire d'avoir un mot pour les chiffres de 0 à 9 et aussi pour les nombres 10,100,1 000. Théoriquement, la combinaison de ces mots permet de dire les nombres de manière régulière sans avoir à créer de nouveau mot (et sans mutation). Mais, selon les langues, le nom des nombres entiers peut faire référence à différentes décompositions du nombre : soit selon les principes de la numération entière, soit en renvoyant à d'autres nombres plus simples. Ainsi, en français, dix-huit évoque dix plus huit unités; quatre-vingts évoque quatre fois le nombre vingt. Notons dans ce domaine les travaux de Guitel (1975) en histoire des mathématiques sur les numérations écrites et ceux de Ascher (1998) dans une perspective ethnomathématique des numérations (mots et symboles).

Le français et le breton proposent ainsi des dénominations qui peuvent être associées à différentes décompositions du nombre. Ceci peut constituer une difficulté : les noms des nombres en breton ne peuvent pas être facilement déduits des noms français, ils doivent être appris. Ceci peut également constituer une ressource : en faisant confronter aux élèves les différents noms de nombres, ceux-ci sont amenés à identifier différentes décompositions du même nombre, ce qui est propice à la conceptualisation.

Par exemple, en breton 18 (mathématiquement $10+8)$ et $50(5 \times 10)$ sont construits sur la base d'autres nombres :

$$
\begin{aligned}
& 18 \text { - triwec' } h \text { - trois-six }-3 \times 6 \\
& 50 \text { - hanter-kant - moitié de cent - 100/2 }
\end{aligned}
$$

Certains noms de nombres correspondent à des décompositions en référence à la base

4 En annexe 2, nous donnons le nom des nombres entiers en langue bretonne et leur traduction littérale an français jusqu'à 2000 . 
vigésimale (base 20 ; il en est de même pour le gallois). Vingt, ugent en breton, permet notamment de construire ${ }^{5}$ :

40 - daou-ugent - littéralement deux-vingts donc mathématiquement $2 \times 20$

60 - tri-ugent - trois-vingts $-3 \times 20$

70 - dek ha tri-ugent - dix et trois-vingts $-10+3 \times 20$

80 - pevar-ugent - quatre-vingts $-4 \times 20$

90 - dek ha pevar-ugent - dix et quatre-vingts $-10+4 \times 20$

Il est donc possible, en particulier pour un professeur, par une comparaison avec le français, de montrer qu'on a ici une continuité dans le principe de construction du nom des nombres pour 40,60 et 80 .

De 11 à 19 (sauf 18), le nom des nombres est du type un-dix $(1+10)$, deux-dix $(2+10)$ donc l'unité en premier suivi du suffixe dix (dek en breton). Cette forme signifie une addition et non une multiplication comme c'est le cas dans d'autres exemples : deux-cents $(2 \times 100)$ en français ou en breton avec daou c'hant (avec la mutation de kant en c'hant). En français, on a cette même construction de onze à seize (avec la mutation du dix en -ze : $1+10, \ldots, 6+10$ ) et ensuite pour dix-sept, dix-huit, dix-neuf, on est sur une forme régulière : 10+7, 10+8, 10+9.

De 21 à 29, war (sur) est utilisé entre les unités et les vingtaines :

\section{1 - unan warn-ugent - un sur-vingt - $1+20$}

22 - daou warn-ugent-deux sur-vingt-2+20, etc.

$H a$ (et) devient la liaison à partir de 31 :

31 - unan ha tregont - un et trente $-1+30$, etc.

Cette différence dans l'ordre des mots peut constituer une ressource pour l'enseignement des mathématiques, ici concernant les propriétés de l'addition. En effet, en breton, 19 se dit neufdix alors que le français se dit dix-neuf, on a donc $10+9=9+10$. A partir de la comparaison des numérations orales en breton et en français, il est donc possible d'envisager un travail en classe sur la commutativité de l'addition.

La comparaison du nom des nombres pourrait constituer une ressource riche pour comprendre le lien entre les numérations orales, les numérations écrites et la modélisation mathématique associée (annexe 2). Cependant celle-ci nécessite des connaissances spécifiques chez les enseignants. Il n'existe pas à notre connaissance un support permettant aux enseignants d'acquérir ces connaissances et comme le montrent les analyses développées ici, un tel support devrait aller bien au-delà d'un simple lexique. On peut parler dans ce cas de « ressource manquante »pour les professeurs des écoles en France (Chevallard \& Cirade 2010). Nous envisageons ce travail de comparaison aussi bien dans les classes monolingues que bilingues. Plusieurs auteurs mettent en évidence une interprétation arithmétique et/ou ordinale des numérations parlée (Cauty 1984, 1986, 1988, puis Mounier 2012). Avec l'usage du mot sur pour la vingtaine, il est possible d'envisager que la numération parlée bretonne soit en partie de type ordinal. Pour notre part, nous choisissons de nous center sur la modélisation mathématique des numérations parlées: A quelle écriture mathématique correspond une numération parlée ? L'étude des similitudes et différences entre plusieurs langues permet un travail mathématique sur la numération et les opérations comme nous montrons ici.

D'autres différences avec le français apparaissent, lorsque l'on aborde « les grands nombres ». Par exemple : 32000 - daou vil ha tregont - deux mille et trente. Ceci est à rapprocher du fait que, en breton, le nom commun exprimé par le nombre s'intercale entre les dizaines et les unités. Ainsi « 32 euros» se dit daou euro ha tregont -deux euros et trente ${ }^{6}$. La figure 2 représente un affichage sur les grands nombres en breton réalisé par un professeur.

5 Nous respectons les règles orthographiques de la langue française pour les traductions littérales. En effet : mille est invariable, vingt et cent s'accordent s'ils ne sont pas suivis d'un déterminant numéral ; et les noms milliers, millions, milliards s'accordent. En breton ugent en invariable.

6 Euro est invariable en breton. En français, pour exprimer les nombres mixtes (ou factionnaires) du type $3 \frac{1}{2}$, l'unité est aussi intercalée entre le nom et la fraction : «trois euros et demis », alors que l'anglais dit « three and a half euros » (Poisard \& Barton 2007). 
Littéralement, 506268483 personnes se lit : « cinq-cent-six millions, deux-cent-huit mille et trois-vingts, quatre-cent-trois personnes et quatre-vingts $\rangle^{7}$. Cette particularité peut être vue comme une difficulté, dans l'apprentissage de la lecture de grands nombres. Les premières analyses de vidéo de classe que nous avons menées laissent toutefois penser que cette particularité de la langue bretonne amène les élèves à réfléchir à la place des chiffres dans un nombre lorsque des grands nombres sont en jeu et permet un travail sur la numération de position. De plus un travail explicite sur cette difficulté : la présence de mille ou million entre l'unité et la dizaine en breton, et non en français, pourrait peut-être permettre de mieux faire prendre conscience aux élèves des groupements de trois chiffres dans les grands nombres.

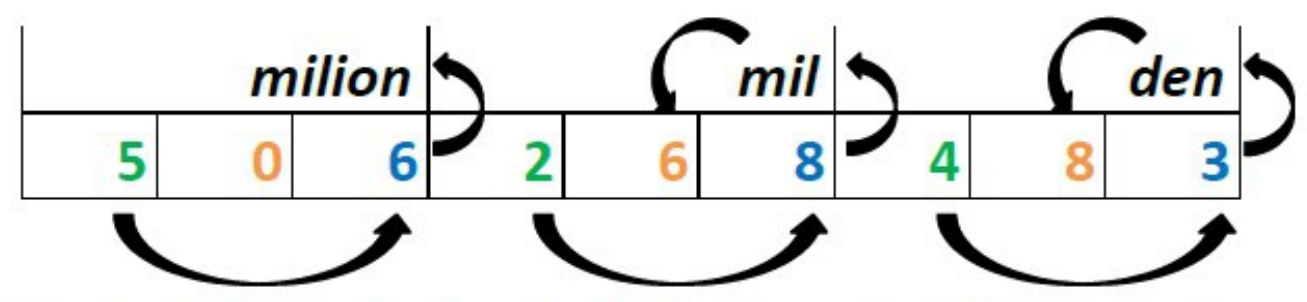

Pemp kant c'hwec'h milion, daou c'hant eizh mil ha tri-ugent, pevar c'hant tri den ha pevar-ugent.

Figure 2. Affichage sur les grands nombres en breton réalisé par un professeur.

Des spécificités de même nature se retrouvent, lorsque l'on aborde les fractions. Ainsi, lorsque le numérateur est supérieur à 20, le dénominateur peut être considéré comme un nom « unité », ce qui donne des formulations telles que :

$76 / 100$ - c'hwezek kantvedenn ha tri-ugent - 16 centièmes et 60

21/10 - un dekvedenn warn-ugent - 1 dixième sur 20

463/857 - pevar c'hant teir eizh kant seizhvedenn ha hanter-kant ha tri-ugent-quatre cent trois huit cent septièmes et demi-cent et trois-vingt

Nous avons pu noter lors d'observations menées en CM2 que nommer des fractions en breton est une tâche délicate pour les élèves, même si ceux-ci nomment correctement les nombres entiers. Certains professeurs nous ont déclaré qu'ils choisissaient pour éviter ces difficultés de traduire littéralement le français en breton. Ils utilisent ainsi une dénomination erronée calquée sur le français : « 463 war 857 » (463 sur 857). En breton ce choix est incorrect, car war signifie sur la quantité, donc en plus. En conséquence, certains professeurs choisissent de dire « 463 rannet dre 857 » (463 divisé par 857). Une telle dénomination conduit à mettre en avant la fraction $a / b$ comme « $a$ divisé par $b$ », par rapport à l'aspect « $a b$-ième » lié au partage d'un objet; mais les professeurs qui effectuent ce choix n'ont pas nécessairement conscience de cette conséquence.

Ceci amène à relativiser la portée des choix terminologiques "officiels », qui ne sont pas nécessairement respectés. Il est cependant intéressant pour notre étude de voir sur quelle base ceux-ci ont été faits.

\subsection{Choix terminologiques}

Selon Barton et alii (1998), la construction d'une terminologie plus évocatrice était l'une des principales retombées positives pour l'enseignement des mathématiques du travail fait en Nouvelle-Zélande sur la langue maorie. Pour la langue bretonne, nous retenons deux mouvements successifs, et opposés, dans les choix de terminologie (nous nous appuyons en particulier ici sur Robin 2001).

7 Ugent et million sont invariables en breton. 
Dans un premier temps (comme dans le cas du maori) ont été retenus des termes évocateurs, utilisant des racines celtiques. Dans un second temps certains termes ont été revus pour se rapprocher du français, en particulier avec l'objectif de faciliter pour les élèves ayant suivi en primaire un enseignement en breton le retour à l'enseignement en français au secondaire (retour qui concerne un nombre d'élèves conséquent, faute, notamment, d'enseignement bilingue à proximité).

C'est le cas notamment pour certains termes de géométrie, comme le terme parallèle. Le glossaire TES de 1998 propose kenstur (et seulement ce terme) qui signifie « de même direction" et est composé du comparatif ken "aussi... que» et de stur qui signifie gouvernail, direction, mouvement. Les termes parallélépipède e t parallélogramme se construisent alors logiquement à partir de kenstur, respectivement kensturdaleg et kensturieg. Le glossaire TES de 2005 marque un changement dans les choix terminologiques avec la mise en place d'un lexique international (Kergoat 2012). En plus des traductions de 1998, apparaissent parallelek gant (parallèle à) et parallelenn (une parallèle) ainsi que parallelepiped et parallelogram. Le passage du terme kenstur à parallelenn s'est effectué dans le glossaire TES mais aussi pour la traduction du manuel pour la classe : J'apprends les maths (traductions éditées entre 1994 et 1998) utilisait le terme de kenstur ; les éditions Cap maths, plus récentes, utilisent parallelenn (pour d'autres exemples, voir Robin 2001).

Ces observations amènent différents constats. D'une part, un professeur peut choisir d'introduire certains concepts en breton, lorsque des termes évocateurs existent (par exemple, périmètre se dit trohed, littéralement longueur du tour), ce qui permet aux élèves un accès plus immédiat au sens du concept ainsi nommé : dans ce cas, la langue se constitue clairement en ressource pour l'enseignement. D'autre part, l'existence de deux termes distincts, pour désigner le même concept, peut amener le professeur à une démarche métalinguistique, enrichissant sa propre réflexion didactique. Nous avons observé un questionnement des professeurs sur les choix terminologiques, pour Loïc par exemple, que nous avons rencontré pour un entretien. Loïc est un professeur des écoles bilingue expérimenté de CM1/CM2 qui a choisi d'enseigner les mathématiques principalement en langue bretonne :

Il y a des choix que je fais, des choix très personnels de vocabulaire, parce qu'on manque de références stables, notamment le vocabulaire scientifique, la biologie ou des choses comme ça. Et là, par exemple pour en revenir au kenstur, et parallelek, qu'on a vus avec les CM1. Dans la méthode de maths que j'utilise depuis presque une dizaine d'années maintenant, ça a toujours été kenstur qui veut dire «le même chemin enfin, dans la même direction, de même direction ». Ce qui a été choisi dans le livre qu'on a eu cette année, qui est donc l'édition 2008, enfin le programme 2008, [...] c'est parallelek qui est la traduction du français, transcription assez proche, c'est une question que je me pose, sachant que c'est beaucoup plus flou au niveau du vocabulaire. Par contre, c'est peut-être beaucoup plus universel. Enfin on est toujours sur cette question-là, on est sur un vocabulaire qui est relativement instable encore... [...] On enlève du culturel pour aller vers de l'universel. Après, pourquoi pas ?

(Entretien avec Loïc)

Notre objectif n'est pas de contribuer au débat sur les terminologies à choisir. Nous retenons de cette partie que des possibilités existent, pour un enseignement en contexte bilingue qui favorise la conceptualisation mathématique, grâce à l'étude de spécificités linguistiques. Les professeurs exploitent-ils ces possibilités en classe, disposent-ils de ressources matérielles susceptibles de les aider à le faire ? Nous nous penchons maintenant sur ces questions.

\section{Pratiques et ressources des professeurs pour l'enseignement des mathématiques en langue bretonne}

Dans cette partie nous allons nous centrer sur les réponses au questionnaire (annexe 1). Celles-ci doivent naturellement être complétées par des observations de pratiques effectives ; cependant elles nous renseignent au moins partiellement sur l'existant, concernant certains points dont nous avons souligné l'importance ci-dessus (partie 2).

Nous avons reçu 80 réponses complètes au questionnaire. Nous remarquons dans un premier 
temps que presque $39 \%$ proviennent d'enseignants ayant moins de 5 ans d'ancienneté en classe bilingue et ce taux décroît avec l'ancienneté, jusqu'à environ $15 \%$ pour les enseignants ayant plus de 15 ans d'ancienneté. Ceci semble témoigner du développement récent de l'enseignement en breton et des recrutements associés ; toutefois il peut s'agir d'un effet de la méthodologie, le questionnaire en ligne étant un média plus familier aux jeunes collègues.

À propos des spécificités de l'enseignement des mathématiques en breton (figure 3), nous retenons que selon les déclarations des professeurs, c'est dans le domaine «nombres entiers et numération » que l'enseignement en breton amène le plus de modifications.

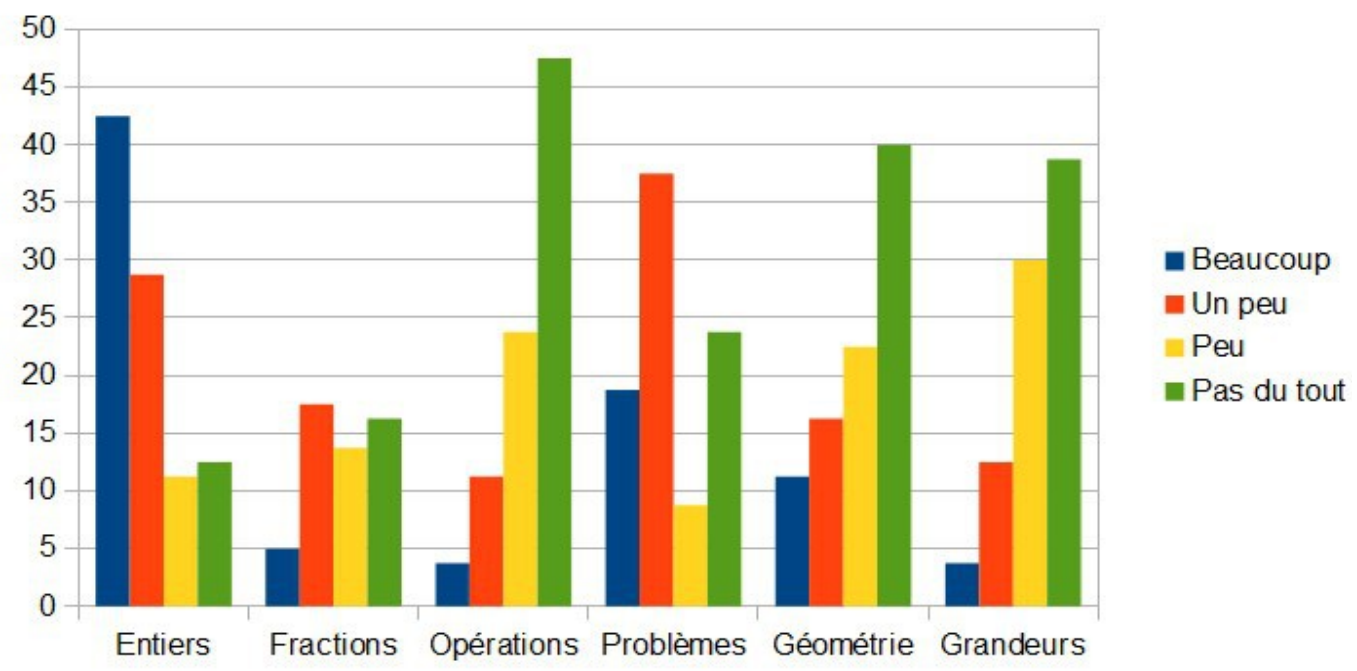

Figure 3: Pourcentages de réponses à la question 2.1: «Est-ce que selon vous l'enseignement des mathématiques en breton modifie l'approche de certains concepts ?»

Ainsi $43 \%$ des professeurs déclarent que l'enseignement en breton modifie " beaucoup » l'approche de la numération entière. Ce constat est fait de façon croissante suivant l'ancienneté des professeurs; parmi les collègues les plus expérimentés (ancienneté supérieure à 15 ans), plus de $72 \%$ (soit 8 professeurs sur les 11) répondent ainsi « beaucoup ». Nous retenons qu'il ne s'agit donc pas d'une préoccupation de début de carrière, mais d'une question intrinsèque : ceci confirme les analyses que nous avons faites ci-dessus (partie 3), concernant le nom des nombres. Pour la " résolution de problèmes », les réponses sont en faveur d'une influence et ce n'est pas l'ancienneté dans l'enseignement bilingue qui a ici son importance mais plutôt le cycle ; en effet du cycle 1 au cycle 3 on voit les réponses en faveur d'une nette influence progresser. Nous faisons l'hypothèse que ceci résulte de la part de l'oral et de l'écrit dans chaque cycle en résolution de problèmes. En effet, en cycle 1, la situation problème et les recherches d'hypothèses sont bien sûr oralisées. Pour le cycle 2 , la prise en compte du support écrit est accompagnée d'explications orales. En revanche, en cycle 3 , et en particulier dans un contexte de gestion du multi-niveaux, les élèves travaillent souvent en autonomie et se retrouvent donc seuls face à un support écrit.

En ce qui concerne les éléments qui influencent la compréhension des concepts (question 2.2), le «vocabulaire» s'avère être mentionné globalement comme un élément nettement déterminant $(85 \%$ de réponses «beaucoup » ou "un peu»). Cependant, cette influence ressentie, tout en restant importante, diminue en fonction de l'ancienneté des professeurs. Cela peut se comprendre par le fait que, pour les enseignants les plus expérimentés, le vocabulaire mathématique en breton ne présente pas de difficulté. Ces enseignants considèrent en grande majorité que le niveau de leurs élèves en breton n'engendre "aucune » ou "peu » de difficultés en mathématiques. Parmi ces enseignants expérimentés, plus de $36 \%$ indiquent un apport important de l'utilisation des deux langues pour l'enseignement des mathématiques, 
c'est-à-dire, selon la perspective retenue ici, considèrent le bilinguisme comme une ressource pour l'enseignement des mathématiques (ce taux n'est que de $16 \%$ pour l'ensemble des répondants). En ce qui concerne la dimension culturelle, seulement $20 \%$ des enseignants considèrent qu'elle peut avoir une influence (beaucoup, ou un peu). Il semble que les enseignants fassent peu de lien entre dimension culturelle et mathématiques.

A propos des difficultés potentiellement engendrées par le niveau en breton des élèves, les professeurs ayant plus de 15 ans d'ancienneté (effectif de 11) disent à $46 \%$ que cela n'engendre aucune difficulté. Les réponses globales sont plus modérées, $76 \%$, indiquant que cela engendre " quelques difficultés», ou « peu de difficultés » et si on observe les réponses en fonction des cycles, on obtient le même type de constat. Il n'y a que les collègues ayant moins de 5 ans d'ancienneté qui pensent que cela engendre «beaucoup de difficultés »: on peut donc supposer qu'au fil de leur pratique, de la formation dont ils peuvent bénéficier en breton tout au long de leur carrière (sur le plan personnel ou au cours de formations institutionnelles), les collègues construisent des compétences et sont en mesure d'apporter des éléments de réponse aux difficultés rencontrées.

En ce qui concerne les ressources (question 3), celles qui semblent les plus utilisées sont les « manuels scolaires en breton »: $60 \%$ des professeurs les utilisent souvent ou régulièrement (viennent ensuite les manuels en français que les professeurs traduisent eux-mêmes, $43 \%$ le font souvent ou régulièrement). Le manuel reste dans tous les cas la ressource centrale, les autres ressources citées étant semble-t-il nettement moins utilisées (" sites web » et «jeux» apparaissent d'une façon qui reste marginale). Nous nous centrons donc ici sur les résultats concernant le manuel.

Nous notons tout d'abord que l'emploi important du manuel en breton (donc traduit, puisqu'actuellement il n'existe aucun manuel directement écrit en breton) peut contribuer à expliquer le peu d'importance accordée par les professeurs aux dimensions culturelles : en effet, ces manuels traduits n'abordent pas ces dimensions.

Concernant les usages du manuel traduit, la situation apparaît assez contrastée, selon les caractéristiques professionnelles des professeurs. Les enseignants de cycle 1 déclarent qu'ils manquent de manuels (ou fichiers) traduits en breton. Pour les autres cycles, le manuel élève Cap Maths est souvent cité par les professeurs dans leurs commentaires, le seul traduit (ou en cours de traduction pour certains niveaux) qui est conforme aux instructions officielles en vigueur. Le livre du maître n'a pas été traduit, ce qui conduit certains collègues à le traduire eux-mêmes pour ce qui touche les activités régulières de calcul mental notamment, mais aussi pour suivre le déroulement proposé pour les situations présentées dans le guide du maître. Autre difficulté, à propos de Cap Maths : ce manuel, issu de la recherche en didactique, propose des situations riches dont la gestion peut être complexe dans le cadre d'un multiniveaux tant dans un contexte bilingue que monolingue. L'équilibre horaire (enseignement immersif, bilingue à mi-temps ou autre) influence également les avis des professeurs à propos des manuels en breton: "Les manuels en breton ne sont pas adaptés au niveau de breton des classes bilingues ${ }^{8}$ qui est faible » déclare ainsi un enseignant. Une telle remarque pose question. D'une part, certains travaux en particulier dans le domaine de l'intercompréhension entre les langues montrent que les élèves peuvent s'engager dans la résolution d'un problème de mathématiques formulé dans une langue étrangère qu'ils ne maîtrisent pas, sans que ceci ne crée un obstacle majeur (Escudé 2010). De plus, le choix de l'enseignement des mathématiques en breton vise aussi à la construction de compétences linguistiques. Si on emploie une langue simplifiée dans cet enseignement, on risque de négliger cet objectif.

8 Cet enseignant fait référence aux classes paritaires, selon la terminologie que nous avons retenue ici. 
Les réponses à la question concernant les besoins des enseignants (question 5) complètent les constats précédents : les enseignants répondent massivement «tout à fait d'accord» ou "plutôt d'accord» pour chaque besoin suggéré (formation continue, échanges avec des collègues, exemples d'activités, logiciels éducatifs, vidéos de classe, conseils de mise en œuvre, glossaires, voir figure 4).

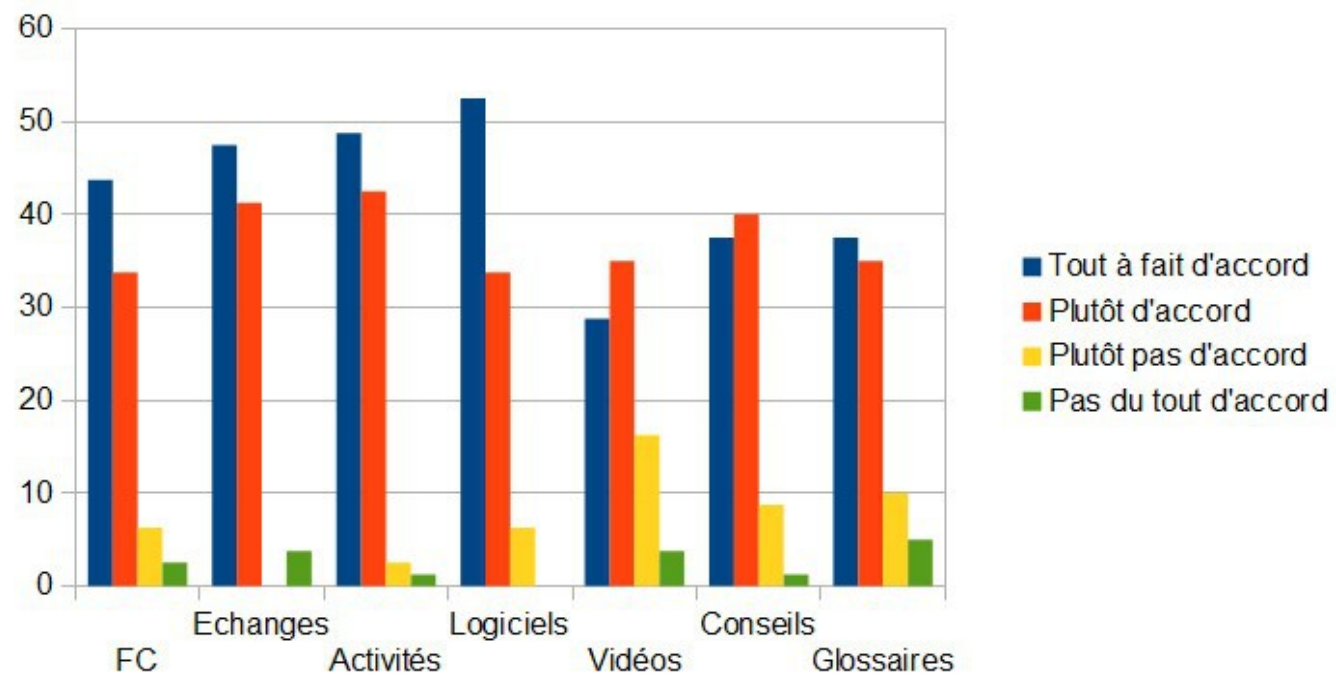

Figure 4. Pourcentages de réponses à la question 5 « Pour améliorer votre enseignement des mathématiques en breton, vous auriez besoin de?»

Ceci est particulièrement vrai pour les échanges avec les collègues, les exemples d'activités, et les logiciels éducatifs (avec plus de $86 \%$ de « tout à fait d'accord » ou " plutôt d'accord »); le type de ressource recueillant le moins de demandes étant les "vidéos de classe ». Les collègues moins expérimentés sont ceux qui demandent le plus d'exemples d'activités, ce qui est probablement une conséquence du fait qu'ils ne disposent pas d'un répertoire déjà précédemment élaboré. Dans les commentaires des sondés, on retrouve des demandes concernant les manuels traduits en breton, le manque de variété de l'offre étant souligné : « Divers manuels traduits plutôt que de faire le choix d'un seul manuel et de le donner dans toutes les classes bilingues (J'apprends les maths avant, Cap maths maintenant) ». Il y a également des demandes de nature plus didactique, non spécifiquement liées à la langue, comme « des logiciels de soutien pour les élèves en difficulté ».

Nous allons ci-dessous revenir sur les questions posées au départ de notre travail, et montrer comment les résultats obtenus permettent d'y apporter des éléments de réponses.

\section{Conclusion}

Les questions posées au départ de notre travail étaient les suivantes :

「 Quels sont les atouts et obstacles potentiels de la langue bretonne comme ressource pour l'enseignement et l'apprentissage des mathématiques ? Il s'agit d'identifier les spécificités de la langue bretonne qui sont susceptibles d'influencer l'apprentissage des mathématiques dans cette langue, ou dans un contexte bilingue breton-français.

「 Est-ce que les ressources matérielles disponibles pour l'enseignement des mathématiques sont susceptibles d'aider les professeurs à exploiter la langue, et la dimension culturelle, pour leur enseignement des mathématiques ?

「Quelles sont les ressources matérielles effectivement utilisées par les professeurs du 
premier degré pour leur enseignement de mathématiques en langue bretonne ? Est-ce que certaines ressources leur manquent ? Est-ce que les professeurs mobilisent effectivement, dans leurs pratiques, la langue bretonne et les dimensions culturelles comme ressources en mathématiques?

Nous avons identifié de véritables potentialités de la langue bretonne. D'une part, celle-ci offre dans certains cas un vocabulaire plus explicite sur le sens des concepts (comme dans le cas de kennstur, pour " parallèle », ou trohed, pour " périmètre »). D'autre part, un travail explicite sur certaines différences linguistiques peut éclairer, en particulier, les propriétés mathématiques fondant la numération entière. Il est possible que d'autres potentialités existent, par exemple en ce qui concerne le calcul mental ; nos observations de classes, qui se poursuivent, devraient permettre de compléter ce que nous avons présenté ici.

Cependant, les ressources disponibles pour les professeurs restent limitées. La ressource la plus utilisée est le manuel en français traduit en breton. Nous avons souligné les spécificités de la langue bretonne, et montré que les difficultés mathématiques ne se situaient pas nécessairement au même endroit en breton et en français. Une traduction d'un manuel peut difficilement respecter ces spécificités. De plus une telle traduction ne peut pas aider les professeurs à tirer parti des potentialités du bilinguisme. En effet, un manuel traduit ne contient ni travail de comparaison linguistique, ni références culturelles.

Or, si nous avons observé des potentialités du bilinguisme, nous avons aussi noté que leur exploitation requiert des compétences spécifiques - pour les élèves comme pour les professeurs. Dans le contexte de notre étude, presque tous les élèves apprennent le breton à l'école. Dans certains cas (enseignement paritaire), ils rencontrent des difficultés avec cette langue, qui peut donc difficilement constituer une aide dans l'apprentissage des mathématiques. Du point de vue des professeurs, il est aussi nécessaire que ceux-ci aient une bonne maitrise du breton. Mais ils doivent également développer des compétences en didactique des mathématiques, pour anticiper les représentations qui peuvent être construites par les élèves en fonction des caractéristiques de la langue utilisée. Nous l'avons observé pour les fractions : il est nécessaire de connaître la distinction entre les points de vue «a b-ième » d'une part, " $\mathrm{a}$ divisé par $\mathrm{b}$ » d'autre part pour comprendre que désigner la fraction $a / b$ par l'expression «a divisé par $b$ » n'est pas neutre. Il faut naturellement, de plus, que les enseignants aient une réflexion sur l'apport des analyses métalinguistiques.

Compétences linguistiques et mathématiques sont indissociables tant au niveau des apprentissages de l'élève que dans la conception à la fois des situations d'apprentissage et des supports d'enseignement. Dans le cas de l'enseignement des mathématiques en breton, ce fait n'est pas encore suffisamment pris en compte. Il est nécessaire de concevoir et diffuser des ressources, en particulier aux enseignants nouvellement formés (en nombre croissant ces dernières années), afin qu'ils soient en mesure d'aborder eux-mêmes des activités de comparaison métalinguistiques avec leurs élèves, concernant les spécificités de la langue bretonne. Le manque de ressources adaptées au contexte des classes bilingues, qui sont de plus très souvent des classes multi-niveaux, ressort clairement du questionnaire. Notre étude souligne la nécessité de créer des ressources permettant un apprentissage à la fois des concepts mathématiques, des spécificités linguistiques de la langue bretonne et de sa dimension culturelle dans un contexte impliquant des phases répétées et alternées en autonomie pour les élèves du fait du multi-niveaux.

Nous allons poursuivre nos recherches, et approfondir l'étude présentée ici en analysant des entretiens avec les enseignants et des vidéos de classe, pour préciser ce que sont les pratiques réelles. Dans le même temps, nous tenterons de contribuer à la production de ressources permettant aux professeurs d'exploiter les potentialités riches que nous avons observées, en 
tenant compte de la complexité des contraintes auxquelles ils sont confrontés.

\section{Références}

Adler J. (2000) "Conceptualising resources as a theme for teacher education 》-Journal of Mathematics Teacher Education 3, (205-224).

Adler J. (2001) Teaching mathematics in multilingual classrooms. Dordrecht : Kluwer Academic Publishers.

Ascher M. (1998) Mathématiques d'ailleurs. Nombres, formes et jeux dans les sociétés traditionnelles. Paris : Seuil.

Barton B. (2008) The language of mathematics. Telling mathematical tales. New York : Springer.

Barton B., Fairhall U. \& Trinick T. (1998) «Tikanga Reo Tatai: Issues in the Development of a Maori Mathematics Register »-For The Learning of Mathematics 18, 1 (3-9).

Broudic F. (2009) Parler breton au XXIe siècle. Brest : Emgleo Breiz.

Brousseau G. (1998) Théorie des situations didactiques. Grenoble : La Pensée Sauvage.

Brousseau G. (2012) «Suggestions d'un didacticien au CS de la conférence»-Conférence nationale sur l'enseignement des mathématiques, Institut Français de l'Education, Lyon. http://educmath.enslyon.fr/Educmath/dossier-manifestations/conference-nationale/contributions/conference-nationale--brousseau-1

Cauty A. $(1984,1986)$ «Taxinomie, syntaxe et économie des numérations parlées »-Amérindia 9\&11.

Cauty A. (1988) « Sémantique de la mise en signes du nombre : une vision ordinale »-Amérindia 13.

Chevallard Y. \& Cirade G. (2010) «Les ressources manquantes comme problème professionnel » - in : G. Gueudet et L. Trouche (éds.) Ressources vives. Le travail documentaire des professeurs en mathématiques (41-55). Rennes/ Lyon : Presses Universitaires de Rennes / INRP.

Cummins J. (1984) Bilingualism and special education. Clevedon : Multilingual Matters.

Cummins J. (2001) Language, Power and pedagogy: bilingual children in the Crossfire. Clevedon : Multilingual Matters.

Escudé P. (2010) Le point sur l'intercompréhension, clé du plurilinguisme. Paris : Clé international.

Gajo L. (2001) Immersion, bilinguisme et interaction en classe. Paris : Didier.

Gajo L. (2007) « Enseignement d'une DNL en langue étrangère : de la clarification à la conceptualisation ». Trema 28, (37-48).

Gueudet G. \& Trouche L. (2010) Ressources vives. Le travail documentaire des professeurs en mathématiques. Rennes / Lyon : Presses Universitaires de Rennes / INRP.

Guitel G. (1975). Histoire comparée des numérations écrites. Paris : Flammarion.

Kergoat L. (2012) "Terminologie bretonne : rétrospective, débats et enjeux 》-Revue RECERC 5, (1-6).

Le Pipec E. (2014, sous presse) « Le breton, langue-totem ? » - La Bretagne linguistique 18.

Margolinas C. (2002) «Situations, milieux, connaissances. L'activité du professeur » - in : J.-L. Dorier, M. Artaud, M. Artigue, R. Berthelot, et R. Floris (éds.) Actes de la XIème école d'été de didactique des mathématiques, (141-155). Grenoble : La Pensée Sauvage.

Meaney T., Fairhall U. \& Trinick T. (2007). « Acquiring the Mathematics Register in te reo Mäori. » - MERGA 30, (493-502).

Mohamed-Sagayar M. (2007) «Analyse de l'Action du professeur : étude didactique d'une séance de classe centrée sur l'enseignement du nombre 80 dans une classe ordinaire de cours préparatoire au Niger. » - in: I. Bloch et F. Conne (éds.) Actes de la XIVème école d'été de didactique de mathématiques. Grenoble : La Pensée Sauvage.

Moschkovich J. N. (2002) «A situated and sociocultural perspective on bilingual mathematics learners »Mathematical Thinking and Learning, Special issue on Diversity, Equity, and Mathematical Learning 4, 2\&3 (189-212).

Moschkovich J. N. \& Nelson-Barber S. (2009) « What mathematics teachers need to know about culture and language » - in : B. Greer, S. Mukhopadhyay, S. Nelson-Barber and A. Powell (éds.) Culturally Responsive Mathematics Education (111-136). New York: Routledge, Taylor \& Francis Group.

Mounier E. (2012). « Des modèles pour les numérations orales indo-européennes à usage didactique. Application à la numération parlée en France. »-Annales de didactique et de sciences cognitives 17, (27-58).

Ní Ríordáin M. (2013) «A comparison of irish and english language features and the potential impact on mathematical processing. » - in : B. Ubuz, C. Haser, et M. A. Mariotti (eds.) Proceedings of CERME 8 (15761585). Ankara : Middle East Technical University. 
Ní Ríordáin M. \& McCluskey A. (2012) « The influence of bilingualism on mathematical problem solving at third level education in Ireland »- in : Proceedings of ICME 12. Seoul, South Korea.

OPLB (Office Public de la Langue Bretonne) (2013) L'enseignement bilingue en 2012. Rapport annuel en ligne : http://www.ofis-bzh.org.

Poisard C. \& Barton B. (2007) «L'utilisation et l'enseignement des fractions en France et en NouvelleZélande.» - in : I. Bloch et F. Conne (éds.) Actes de la XIVème école d'été de didactique de mathématiques. Grenoble : La Pensée Sauvage.

Robin J.-D. (2001) «L'enseignement (des mathématiques) en breton est-il une gageure ? » -Klask 7, (78-98).

Setati M. \& Planas N. (2012) «Mathematics education across two different language contexts: A political perspective » - in : O. Skovsmose et B. Greer (éds.) Opening the Cage: Critique and Politics of Mathematics Education (167-186). Rotterdam: Sense Publishing.

Swain M. (1996) « Integrating language and content in immersion classrooms: Research prospective »-The

Canadian Modern Language Review 52, 4 (529-54).

Vygotsky L. (1978) Mind in society. Cambridge, MA: Harvard University Press. 


\section{Annexe 1 : Questionnaire « mathématiques en langue bretonne »}

\section{Description de vos conditions d'enseignement des mathématiques en breton}

Depuis combien de temps êtes-vous enseignant en classe bilingue ?

Comment avez-vous appris le breton ?

Parlez-vous breton?

- depuis votre enfance

- depuis vos études

- autres

Quelle classe avez-vous en 2012-2013?

N.B. Dans la suite du questionnaire, la dénomination « vos élèves » se réfère à cette classe

Combien d'heures consacrez-vous chaque semaine à l'enseignement des mathématiques en breton?

Est-ce que vous enseignez également les maths en français aux mêmes élèves ?

$\mathrm{Si}$ oui, avec quel équilibre horaire entre les deux langues?

\section{Enseignement des mathématiques en breton}

2.1 Est-ce que selon vous, l'enseignement des mathématiques en breton modifie l'approche de certains concepts?

\begin{tabular}{|l|l|l|l|l|}
\hline & beaucoup & un peu & peu & pas du tout \\
\hline $\begin{array}{l}\text { Nombres entiers et } \\
\text { numération }\end{array}$ & & & & \\
\hline Fractions et décimaux & & & & \\
\hline Opérations & & & & \\
\hline Résolution de problèmes & & & & \\
\hline Géométrie & & & & \\
\hline Grandeurs et mesures & & & & \\
\hline
\end{tabular}

Commentaires éventuels :

2.2 Quels sont, d'après vous les éléments qui peuvent influer sur la compréhension des concepts mathématiques lorsqu'ils sont enseignés en breton

\begin{tabular}{|l|l|l|l|l|}
\hline & influence beaucoup & influence un peu & influence peu & n'influence pas du tout \\
\hline Le vocabulaire & & & & \\
\hline $\begin{array}{l}\text { L'utilisation de } \\
\text { deux langues }\end{array}$ & & & & \\
\hline $\begin{array}{l}\text { La dimension } \\
\text { culturelle }\end{array}$ & & & & \\
\hline
\end{tabular}

2.3 Considérez vous que le niveau de vos élèves en breton engendre des difficultés pour votre enseignement des mathématiques?

\begin{tabular}{|l|c|c|c|c|}
\hline & $\begin{array}{c}\text { engendre beaucoup } \\
\text { les difficultés }\end{array}$ & $\begin{array}{c}\text { engendre quelques } \\
\text { difficultés }\end{array}$ & $\begin{array}{c}\text { engendre peu de } \\
\text { difficultés }\end{array}$ & $\begin{array}{c}\text { n'engendre aucune } \\
\text { difficulté }\end{array}$ \\
\hline $\begin{array}{l}\text { Le niveau de mes } \\
\text { élèves en breton }\end{array}$ & & & & \\
\hline
\end{tabular}

\section{Ressources}

Pour préparer vos séances de mathématiques en breton est-ce que vous :

\begin{tabular}{|l|l|l|l|l|}
\hline & rarement & parfois & régulièrement & souvent \\
\hline utilisez des manuels scolaires en breton & & & & \\
\hline utilisez des manuels en français que vous traduisez & & & & \\
\hline utilisez des sites web en breton & & & & \\
\hline $\begin{array}{l}\text { travaillez avec des collègues de maths des classes } \\
\text { monolingues }\end{array}$ & & & & \\
\hline $\begin{array}{l}\text { utilisez des ressources en d'autres langues que le } \\
\text { français et le breton }\end{array}$ & & & & \\
\hline
\end{tabular}

Autres ressources :

Commentaires éventuels : 


\section{Liens avec l'enseignement des mathématiques en français}

Si vous enseignez aussi les mathématiques en français, votre enseignement en breton influence-t-il votre enseignement en français?

\begin{tabular}{|c|c|c|c|}
\hline beaucoup & un peu & peu & pas du tout \\
\hline & & & \\
\hline
\end{tabular}

Commentaires éventuels :

\section{Besoins identifiés}

Pour améliorer votre enseignement des mathématiques en breton, vous auriez besoin de :

\begin{tabular}{|l|l|l|l|l|}
\hline & $\begin{array}{c}\text { tout à fait } \\
\text { d'accord }\end{array}$ & plutôt d'accord & plutôt pas d'accord & $\begin{array}{c}\text { pas du tout } \\
\text { d'accord }\end{array}$ \\
\hline Formation continue & & & & \\
\hline Échanges avec des collègues & & & & \\
\hline Exemples d'activités & & & & \\
\hline Supports pour la langue (glossaires) & & & & \\
\hline Vidéos de classe & & & & \\
\hline Logiciels éducatifs & & & & \\
\hline Conseils de mise en oeuvre & & & & \\
\hline
\end{tabular}

Autres besoins :

Commentaires éventuels :

\section{Merci de votre contribution}




\section{Annexe 2 : Analyse des numérations orales en breton et en français}

\section{Le nom des nombres en langue bretonne (jusqu'à 2 000)}

Pour chaque nombre, nous proposons la traduction littérale en français et la modélisation mathématique associée. Nous respectons les règles orthographiques de la langue française pour les traductions littérales. En effet : mille est invariable, vingt et cent s'accordent s'ils ne sont pas suivis d'un déterminant numéral ; et les noms milliers, millions, milliards s'accordent. Pour le breton, les nombres ne sont jamais au pluriel (sauf si on utilise la préposition $a$ ). Donc ugent, kant (c'hant), mil (vil), million sont invariables. De plus, le breton utilise le tiret différemment du français.

\section{Breton}

Traduction littérale en français

zero (Il existe le terme mann qui signifie rien qui est peu employé.)

unan

daou / div (masculin/féminin)

tri / teir

pevar / peder

pemp

c'hwec'h

seizh

eizh

nav

dek

unnek (mutations de unan et dek)

daouzek (mutation de dek)

trizek (mutation de dek)

pevarzek (mutation de dek)

pemzek (mutations de pemp et dek)

c'hwezek (mutations de $c^{\prime} h w e c^{\prime} h$ et dek)

seitek (mutations de seizh et dek)

triwec'h

naontek (mutations de nav et dek)

$\begin{array}{ll}\text { un-dix } & 1+10 \\ \text { deux-dix } & 2+10 \\ \text { trois-dix } & 3+10 \\ \text { quatre-dix } & 4+10 \\ \text { cinq-dix } & 5+10 \\ \text { six-dix } & 6+10 \\ \text { sept-dix } & 7+10 \\ \text { trois-six } & \mathbf{3} \times \mathbf{6} \\ \text { neuf-dix } & 9+10\end{array}$

ugent

unan warn ugent

un-sur-vingt $\quad 1+20$

daou warn ugent

deux-sur-vingt $\quad 2+10$

tregont

unan ha tregont

un-et-trente

$1+30$

40 daou-ugent

41 unan ha daou-ugent

\section{hanter-kant}

tri-ugent

dek ha tri-ugent

pevar-ugent

dek ha pevar-ugent

deux-vingts $2 \times 20$

un-et-deux-vingts $1+2 \times 20$

moitié de cent $\quad 100 / 2$

trois-vingts $3 \times 20$

dix-et-trois-vingts $\quad 10+3 \times 20$

quatre-vingts $\quad 4 \times 20$

dix-et-quatre-vingts $\quad 10+4 \times 20$

kant

200 daou c'hant (mutation de kant en c'hant)

cent

100

deux-cents $2 \times 100$

trois-cents $3 \times 100$

pevar c'hant (mutation de kant en $c^{\prime}$ hant)

quatre-cents

$4 \times 100$

pemp kant

c'hwec'h kant

cinq-cents

$5 \times 100$

six-cents

$6 \times 100$

seizh kant

eizh kant

sept-cents

$7 \times 100$

huit-cents

$8 \times 100$

nav c'hant (mutation de kant en c'hant)

neuf-cents

$9 \times 100$

$1000 \mathrm{mil}$

2000 daou vil (mutation de mil en vil)

mille

1000

deux-mille $\quad 2 \times 1000$ 


\section{Le nom des nombres en langue française (jusqu'à 2000 ) en France}

Pour chaque nombre, nous proposons la modélisation mathématique associée.

Nous appelons comptine régulière une comptine dont les noms des nombres correspondent à la signification mathématique sous-jacente (décomposition polynomiale).

Nous respectons les règles orthographiques de la langue française. En effet : mille est invariable, vingt et cent s'accordent s'ils ne sont pas suivis d'un déterminant numéral; et les noms milliers, millions, milliards s'accordent. Dans un contexte historique, il n'est pas rare de dire, quinze-cent-quinze pour 1515 , donc d'effectuer des groupements par deux chiffres (et non trois). Qui connaît la célèbre bataille de mille-cinq-cent-quinze ?

\section{Français}

zéro

un / une

deux

trois

quatre

cinq

six

sept

huit

neuf

0 dix

11 onze (mutations de $u n$ et dix)

12

13

14

15

16

17

18

19

20

21

22

…

30

40

50

60

70

80

90

100

200

douze (mutations de deux et dix)

treize (mutations de trois et dix)

quatorze (mutations de quatre et dix)

quinze (mutations de cinq et dix)

seize (mutations de six et $d i x$ )

dix-sept

dix-huit

dix-neuf

vingt

vingt-et-un*

vingt-deux

trente

quarante

cinquante

soixante

soixante-dix

$60+10$

quatre-vingts

quatre-vingt-dix

cent

deux-cents

1000 mille

2000 deux-mille

\section{Comptine régulière}

$\begin{array}{ll}\text { (un-)dix } & 1 \times 10 \\ \text { (un-)dix-et-un } & 1 \times 10+1 \\ \text { (un-)dix-et-deux } & 1 \times 10+2 \\ \text { (un-)dix-et-trois } & 1 \times 10+3 \\ \text { (un-)dix-et-quatre } & 1 \times 10+4 \\ \text { (un-)dix-et-cinq } & 1 \times 10+5 \\ \text { (un-)dix-et-six } & 1 \times 10+6 \\ \text { (un-)dix-et-sept } & 1 \times 10+7 \\ \text { (un-)dix-et-huit } & 1 \times 10+8 \\ \text { (un-)dix-et-neuf } & 1 \times 10+9 \\ & \\ \text { deux-dix } & 2 \times 10 \\ \text { deux-dix-et-un } & 2 \times 10+1 \\ \text { deux-dix-et-deux } & 2 \times 10+2 \\ & \\ \text { trois-dix } & 3 \times 10 \\ \text { quatre-dix } & 4 \times 10 \\ \text { cinq-dix } & 5 \times 10 \\ \text { six-dix } & 6 \times 10 \\ \text { sept-dix } & 7 \times 10 \\ \text { huit-dix } & 8 \times 10 \\ \text { neuf-dix } & 9 \times 10 \\ & \\ \text { un-cent } & 1 \times 100 \\ \text { deux-cents } & 2 \times 100 \\ & \\ \text { un-mille } & 1 \times 1000 \\ \text { deux-mille } & 2 \times 1000\end{array}$

* En français, le mot et existe entre le rang des dizaines et des unités pour 21, 31, 41, 51, 61 et aussi dans 71. 